\title{
Reward Processing under Chronic Pain from the Perspective of "Liking" and "Wanting": A Narrative Review
}

\author{
Xinhe Liu, ${ }^{1,2}$ Ning Wang $\mathbb{D}^{1,2}$ Lijia Gu, ${ }^{1,2}$ Jianyou Guo $\mathbb{D}^{1,2}$ Jinyan Wang $\mathbb{D},{ }^{1,2}$ \\ and Fei Luo iD ${ }^{1,2}$ \\ ${ }^{1}$ CAS Key Laboratory of Mental Health, Institute of Psychology, Chinese Academy of Sciences, Beijing 100101, China \\ ${ }^{2}$ Department of Psychology, University of Chinese Academy of Sciences, Beijing 100049, China \\ Correspondence should be addressed to Ning Wang; wangn@psych.ac.cn
}

Received 3 January 2019; Revised 6 March 2019; Accepted 4 April 2019; Published 21 April 2019

Academic Editor: Monika I. Hasenbring

Copyright (c) 2019 Xinhe Liu et al. This is an open access article distributed under the Creative Commons Attribution License, which permits unrestricted use, distribution, and reproduction in any medium, provided the original work is properly cited.

\begin{abstract}
The therapeutic goals of patients with chronic pain are not only to relieve pain but also to improve the quality of life. Chronic pain negatively affects various aspects of daily life, such as by decreasing the motivation to work and reward sensitivity, which may lead to difficulties in daily life or even unemployment. Human and animal studies have shown that chronic pain damages reward processing; the exploration of associated internal mechanisms may aid the development of treatments to repair this damage. Incentive salience theory, used widely to describe reward processing, divides this processing into "liking" (reward-induced hedonic sensory impact) and "wanting" (reward-induced motivation) components. It has been employed to explain pathological changes in reward processing induced by psychiatric disorders. In this review, we summarize the findings of studies of reward processing under chronic pain and examine the effects of chronic pain on "liking" and "wanting." Evidence indicates that chronic pain compromises the "wanting" component of reward processing; we also discuss the neural mechanisms that may mediate this effect. We hope that this review aids the development of therapies to improve the quality of life of patients with chronic pain.
\end{abstract}

\section{Introduction}

Chronic pain is a worldwide problem for which effective treatment is lacking [1]. It is associated with physical disability, cognitive impairment, and negative psychological states, including anxiety, depression, and an increased risk of suicide [2-4]. Thus, chronic pain is composed of long-term physical and psychological pain, and an important goal of its treatment is to improve patients' quality of life [5]. Chronic pain-induced psychological changes may interfere with the processing of rewards, defined as "objects or events that generate approach and consummatory behavior, produce learning of such behavior, represent positive outcomes of economic decisions and engage positive emotions and hedonic feelings" (page 1, second paragraph) [6]. Given this definition, reward processing has several components, including the noticing of reward-related cues, bursts of motivation, hedonic perception, and reinforcement learning $[7,8]$. Patients with chronic pain may lose pleasure in life and/or motivation to work $[9,10]$, decreasing their sensitivity to rewards [11], but the mechanism by which chronic pain affects reward processing remains unclear. Exploring changes in reward processing under chronic pain may facilitate the improvement of treatment.

Researchers have used the incentive salience theory to explain reward processing during depression. This approach divides reward processing into "liking" and "wanting," both of which are decreased under major depression [12-14]. "Liking" is the pleasurable experience derived from sensory input, usually dependent on reward stimulus' properties, and "wanting" is the internal motivational component related to the acquisition of reward stimuli [15]. In this review, we summarize studies of reward processing during chronic 
pain and discuss related neural mechanisms from the perspective of incentive salience theory.

\section{Reward-Related Behaviors in Patients with Chronic Pain}

2.1. Natural Reward-Related Behaviors during Chronic Pain. Studies conducted with healthy volunteers have shown that acute physical pain can increase the motivation to attain rewards under laboratory conditions [16, 17]. However, natural reward-related behaviors in patients with chronic pain may be more complicated. In a cross-sectional study, Geha and colleagues [11] found reduced hedonic perception of palatable food in patients with CLBP; "liking" ratings for palatable puddings were significantly lower among patients with CLBP than among controls, whereas sensory ratings of intensity, sweetness, creaminess, fattiness, and oiliness, as well as "liking" ratings for sugar-containing drinks, were similar in the two groups. These results indicate that patients with CLBP had normal senses of taste, but decreased foodrelated pleasure. In another study, patients with chronic back pain had lower detection thresholds for gustatory stimuli (i.e., bitter, salty, sweet, and sour) than did controls, with no significant difference in "pleasant" and "unpleasant" ratings for these stimuli between groups [18]. Thus, whether chronic pain affects the derivation of pleasure from gustatory stimuli remains a matter of debate.

2.2. Reward Sensitivity in Patients with Chronic Pain. The term "reward sensitivity" is used to describe positive beliefs about the probability of future rewards [19]. High reward sensitivity helps to identify and motivate repetition of pleasurable activities, whereas low reward sensitivity reduces the motivation to engage in such activities and facilitates the development or aggravation of depressive symptoms [20]. Anhedonia, a typical symptom of depression, is defined as loss of sensitivity to pleasure and motivation to acquire reward $[13,19]$. In early studies, the 66-item Physical Anhedonia Scale (PAS) was used to assess physical anhedonia symptoms in patients with chronic facial and low back pain $[21,22]$. PAS items encompass loss of the ability to feel pleasure from eating, drinking, touching, sex, temperature, smells, and sounds. Thus, the PAS inventory assesses mainly the capacity to hedonically perceive events and stimuli during daily activity. Patients with chronic pain reported high levels of physical anhedonia in these studies, suggesting that chronic pain reduces the emotional pleasure response.

The Behavioral Activation Scale/Behavioral Inhibition Scale (BAS/BIS), based on these behavioral approach system/behavioral inhibition system characteristics [23], has been used to assess reward sensitivity in human subjects. The behavioral approach system is sensitive to reward signals, and the behavioral inhibition system could be activated by aversive stimuli $[19,24]$. Using the BAS/BIS scales, Elvemo et al. [25] found reduced reward responsiveness, defined as tendency to respond positive in the context of desired events or cues of potential future reward, in female patients with chronic idiopathic, visceral, and musculoskeletal pain, but no difference in reward drive, defined as the tendency to pursue reward, between these patients and a control group. They also observed significant higher physical anhedonia in chronic pain patients measured with the Beck Depression Inventory II. Becerra-García and Robles Jurado [24] used questionnaire, based on the behavioral approach system, to assess reward sensitivity in female patients with fibromyalgia, finding significantly reduced behavioral approach system activity, which could be interpreted as poor response to environmental incentives and reduced reward approach behavior. These studies have revealed the correlation between chronic pain and low reward sensitivity.

\subsection{Goal Pursuit Behaviors in Patients with Chronic Pain.} Researchers have examined motivation through assessment of the pursuit of goals [26-29], conceptualized as "internal representations of desired states, where states are broadly construed as outcomes, events, or processes" (Page 338, paragraph 1) [26]. Goal pursuit may be related to reward processing $[30,31]$, and its relationship to chronic pain has been explored using the daily diary method. Hardy et al. asked women with fibromyalgia to record feelings of pain, emotional distress, and fatigue and to rate goal conflicts in daily activity, including household and job tasks and interpersonal relationships, using daily diaries [32]. They found that goal pursuit behaviors resulted in greater subjective feelings of pain and that emotional distress mediated the perception of goal conflicts. Mun et al. used the daily dairy method to assess daily goal conflicts in patients with chronic pain [10], finding that pain intensity was related positively to interference with work goals and that this effect was moderated by pain acceptance. Semistructured interviews have also been used to investigate daily goal conflicts in patients with fibromyalgia, revealing more self-reported pain-induced goal conflicts (most commonly affecting household, social, and interpersonal goals) in these patients than in healthy individuals [28]. These studies show that chronic pain inhibits daily goal pursuit activities. Under chronic pain, some short- and long-term goals may become unattainable [27], which may lead to the loss of positive feedback and attenuate the reward processing of goal pursuit behaviors. However, the direct relationship between reward processing and goal pursuit behaviors during the chronic pain remains to be discussed.

The aforementioned studies are summarized in Table 1. These researchers adopted different experimental paradigms, and the results consistently indicated that chronic pain disrupted various aspects of reward processing. Under some conditions, the effects of chronic pain on the emotional and sensory aspects of reward processing may be separate $[11,18]$. Moreover, chronic pain and its management were found to affect daily activities by inducing more goal conflict, which may lead to the adaptive reduction of reward-seeking motivation. In the following sections, we introduce incentive salience theory and use this perspective to present research on reward processing under chronic pain. As only few human studies have directly explored the relationship between chronic pain and reward processing using incentive 
TABLE 1: Reward-related behaviors in patients with chronic pain.

\begin{tabular}{|c|c|c|c|c|}
\hline Study & Disorders & Test & Measurement & Results \\
\hline \multirow{8}{*}{ Geha et al. [11] } & \multirow{8}{*}{ Back pain } & \multirow{5}{*}{ Rating sugary drinks } & Liking & No change \\
\hline & & & Wanting & No change \\
\hline & & & Sweetness & No change \\
\hline & & & Intensity & No change \\
\hline & & & Liking & Decrease \\
\hline & & \multirow{3}{*}{ Rating fat puddings } & Wanting & No change \\
\hline & & & Sweetness & No change \\
\hline & & & Intensity & No change \\
\hline Small and Apkarian [18] & Low back pain & Rating sucrose solution & Pleasantness & No change \\
\hline Marbach and Lund [21] & Farial nain and TMI nain & & Intensity & Increase \\
\hline Marbach and Lund [21] & Facial pain and $1 M$ ) pain & PAS & Anhedonia & $\begin{array}{c}\text { Increase } \\
\text { anreloted to }\end{array}$ \\
\hline Marbach et al. [22] & Back pain or facial pain & PAS & Anhedonia & $\begin{array}{l}\text { Uncorrelated to } \\
\text { pain intensity }\end{array}$ \\
\hline \multirow{3}{*}{ Elvemo et al. [25] } & \multirow{3}{*}{ Various } & BIS/BAS & Reward drive & No change \\
\hline & & BDI & Anhedonia & Increase \\
\hline & & $\mathrm{BIS} / \mathrm{BAS}$ & RER & Decrease \\
\hline Becerra-Garcia and Robles Jurado [24] & Fibromyalgia & SPSRQ & RER & Decrease \\
\hline Claes et al. [28] & Fibromyalgia & Semistructured interview & Goal conflicts & Increase \\
\hline Hardy et al. [32] & Fibromyalgia & Daily diary & Goal conflicts & Increase \\
\hline Mun et al. [10] & Not mentioned & Daily diary & Goal conflicts & Increase \\
\hline
\end{tabular}

Abbreviations: TMJ, temporomandibular joint; PAS, Physical Anhedonia Scale; BIS, Behavioral Inhibition Scale; BAS, Behavioral Activation Scale; BDI, Beck Depression Inventory; SPSRQ, Sensitivity to Punishment and Sensitivity to Reward Questionnaire; RER, reward-induced emotional responsiveness.

salience theory, we describe here mainly evidence derived from animal research in the following chapters. Although animal studies are not entirely a representative of human studies, they may provide important reference for future human studies and facilitate the understanding of how chronic pain affects reward processing.

\section{Reward Processing during Chronic Pain in Animal Studies}

3.1. Incentive Salience Theory. Incentive salience theory has been applied widely to explain reward processing in individuals with psychiatric disorders, such as drug addiction, gambling disorders, overeating $[15,33,34]$, major depression [12-14], and schizophrenia [35]. Within the framework of this theory, "wanting" can be motivated by reward-conditioned cues, and thus may involve associative learning, attention, and memory retrieval. As "wanting" can be translated into motivation and bursts of reward-seeking behavior (i.e., approach behaviors and attempts to obtain stimuli), some researchers refer to it as "incentive salience" $[15,36,37]$. In the context of the consumption of delicious food, "liking" is the pleasurable emotional experience induced via the activation of peripheral sensory (gustatory, tactile, and/or olfactory) receptors, which triggers nerve impulses and activates the reward circuit [38]; "wanting" is the internal desire and wish to repeat the experience triggered by the smell or sight of the food [15, 39].

"Liking" and "wanting" have been measured using specific experimental paradigms. As the hedonic experience dominates during the consumption of reward stimuli [40], the pleasurable sensory stimulus of sweetness has often been used to measure "liking." The sucrose preference test (SPT) measures the consumption of sucrose-containing water, which could reflect the pleasurable sensory input of this activity in animal studies [13]. Taste reactivity is another classical paradigm used to measure "liking," through the scoring of animals' orofacial expressions and behavior during the consumption of sucrose-containing water; tongue protrusion and paw licking, for example, are regarded as behavioral indices of pleasure [36, 41, 42]. A more diverse set of paradigms is used to measure "wanting," as the degree of motivation to acquire reward stimuli. Experiments have involved reward-seeking or goal pursuit tasks $[15,43]$, such as T-maze navigation for rats [41]. The self-administration (SA) model with natural rewards or addictive drugs has also been used widely to measure "wanting" through quantification of the amount of reward earned in rats (e.g., by lever presses or nose pokes) [44-46]. "Wanting" has also been measured by quantifying rats' daily consumption of food or water $[43,47,48]$.

\subsection{Effects of Chronic Pain on "Liking"-Related Behaviors.} Animal studies of the effect of chronic pain on rewardrelated behaviors are summarized in Table 2. As the decreased preference for sucrose in the SPT is often explained as anhedonia, this test has been used widely to examine the comorbidity of chronic pain and depressive emotion in animal research. Several studies have shown that chronic pain decreases rodents' preference for sucrose-containing water. For example, Dellarole et al. reported that chronic constriction injury (CCI) in mice induced neuropathic pain that decreased the $1 \%$ sucrose-containing water preference, reduced body weight, and degraded the physical state of the rats' coats; this process was combined with neuroplastic remodeling in the hippocampus, a key emotional area [49]. Thus, the authors concluded that CCI induced depressive emotion. Wang et al. reported that spared nerve injury (SNI) 
TABLE 2: Reward-related behaviors in animals with chronic pain.

\begin{tabular}{lcccc}
\hline Study & Species & Reward stimuli & Pain model & Test (results) \\
\hline Dellarole et al. [49] & Mouse & SW & CCI & SPT (decrease) \\
Wang et al. [50] & Rat & SW & SNI & SPT (decrease) \\
Wu et al. [51] & Mouse & SW & SNI (decrease) \\
Bura et al., 2013 & Mouse & SW & PSNL & SPT (decrease) \\
Amorim et al. [52] & Rat & SW & Arthritis & SPT (decrease) \\
Liu et al. [53] & Rat & SW & Fibromyalgia & SPT (decrease) \\
Shi et al. [54] & Rat & SW & SNL & SPT (no change) \\
Shi et al. [55] & Rat & SW & CFA & SPT (no change) \\
Su et al. [56] & Rat & SW & SNI; CFA & SPT (decrease) \\
Urban et al. [57] & Mouse & SW; food; water & SNI; CCI; CFA & SPT (no change); food intake (no change); water \\
Bravo et al. [58] & Rat & Cereals & CCI & Food intake (no change) (no change) \\
Goffer et al. [59] & Rat & SW; water & SNI & SPT (decrease); water intake (no change) \\
Okun et al. [60] & Rat & Food & CFA, SNL & SA with PR (CFA decrease, SNL no change); SA with \\
Schwartz et al. [61] & Mouse & SW; food & CFA (no change); tasty reactivity (no change) \\
Hipólito et al. [62] & Rat & SW & CFA & SPT (no change); SA with PR (decrease); SA with FR \\
Schwartz et al. [63] & Rat & SW & (no change); food intake (no change) \\
\hline
\end{tabular}

Abbreviations: SW, sucrose-containing water; SNI, spinal nerve injury; SPT, sucrose preference test; CCI, chronic constriction injury; CFA, complete Freund's adjuvant injection; SNL, spinal nerve ligation; SA, self-administration; PR, progressive ratio protocol; FR, fixed ratio protocol; PSNL, partial ligation of the sciatic nerve; AAT, avoidance-reward approach task.

induced neuropathic pain that decreased rats' preference for sucrose-containing water [50]. Neuropathic pain has also been found to attenuate the hedonic perception of sweetness in mice $[51,52]$. Reduced sucrose preference has also been observed in other chronic pain models, such as those of monoarthritis [64] and fibromyalgia [53].

Other animal studies employing the SPT have revealed no reduction of sucrose preference during chronic pain $[60,61]$. Urban and colleagues investigated the daily behaviors of mice, including their hedonic perception during the SPT [57]. After SNI or complete Freund's adjuvant (CFA) injection, the animals' preference for water containing $2 \%$ sucrose, daily food and water consumption, and weight remained unchanged for weeks. Shi et al. used the SPT to explore the interaction between chronic pain and depression [54], but they observed that spinal nerve ligation (SNL) alone was not sufficient to decrease rats' preference for water containing $1 \%$ sucrose. In other researches, Shi et al. also reported CFA-induced chronic pain inflammatory is not enough to decrease the sucrose preference [55]. Bravo et al. examined depressive symptoms in rats with CCI and similarly found that neuropathic pain did not affect the preference for sweet cereals, food consumption, or body weight [58].

Thus, results regarding the effects of chronic pain on "liking" are inconsistent. One possible reason is that the SPT is not a stable index and can be influenced by many factors, such as experimental conditions, animal types, and chronic pain models. For example, some researchers performed the SPT within 2 weeks after the induction of chronic pain models $[56,59]$, which may be an insufficient time period to allow for alteration of the "liking" component.
3.3. Effects of Chronic Pain on "Wanting"-Related Behaviors. Chronic pain has shown divergent effects on "wanting" behavior, according to the experimental protocol used (Table 2). Some researchers have adopted SA models to explore the effects of chronic pain on reward-seeking behaviors using fixed ratio (FR) and progressive ratio (PR) protocols. Under the FR protocol, animals must press a lever or perform a nose poke a fixed number of times to earn a reward. Under the PR protocol, the number of actions required to obtain the reward increases, making reward acquisition progressively more difficult. Hipólito and colleagues reported that CFA injectioninduced inflammatory pain did not affect rats' sucrose pellet SA behavior under an FR protocol, but that this behavior was attenuated under a PR protocol with sucrose pellets or $50 \mu \mathrm{g} /$ $\mathrm{kg}$ heroin, but not the seeking behaviors to high dose of heroin $(200 \mu \mathrm{g} / \mathrm{kg})$ [62]. Schwartz et al. trained mice to nose poke to obtain reward pellets and built chronic pain models with CFA injection and SNI, respectively [61]. They observed that these treatments decreased the nose poke behavior to food pellets under a PR protocol, but did not affect nose poke behavior or daily food consumption under an FR protocol. Similarly, Okun et al. observed that CFA injection-induced inflammatory pain transiently decreased SA lever pressing in rats under a PR protocol, whereas SNL did not affect SA behavior or daily water or food consumption under an FR protocol [60].

Decreased reward-seeking motivation under chronic pain has also been demonstrated using the pain avoidancereward approach task. Schwartz et al. [63] trained rats to seek small and large rewards (water containing 3\% and $10 \%$ sucrose, respectively) with the probability of experiencing laser-induced heat pain. The rats maintained SA behavior for 
both sucrose concentrations while ignoring the heat pain attacks. After SNI, neuropathic pain reduced SA behavior for the small reward, but did not affect large reward seeking. These results might be interpreted as reflecting the motivational value of reward decrease under chronic pain.

Differences in the difficulty of animals' reward acquisition among experimental paradigms (i.e., between FR ad $\mathrm{PR}$ protocols) may have led to the divergent results regarding the effect of chronic pain on "wanting" behavior. Most SA studies performed with PR protocols have shown that chronic pain decreases "wanting," whereas those performed with FR protocols have shown no effect on SA behavior or daily food or water intake [57, 58, 59], which may reflect the subjective perception of the effort required to complete the task. Researchers believe that, at a certain breakpoint (BP), animals stop nose poking or lever pressing because they perceive that earning the reward is no longer worth the effort. Thus, the BP has been considered to reflect the internal motivation to obtain the reward, which is measured more suitably with a PR protocol than with an FR protocol or the measurement of spontaneous food and water intake [65]. Therefore, we consider that SA models with PR protocols reflect more advanced "wanting" components, which are much more sensitive to chronic pain.

\section{Modulatory Mechanisms of Chronic Pain on Reward Processing}

Pharmacological activation of the dopamine (DA) system, which is one kind of the neurotransmitter closely related to reward, by amphetamine has been found to enhance food intake ("wanting"), but to have no effect on taste reactivity ("liking") in rats [41, 48, 66, 67]. The role of the opioidergic system in reward processing has also been examined; pharmacological stimulation of mu, delta, and kappa receptors in the anterior-posterior nucleus accumben (NAc, a hedonic "hotspot") increased tasty reactivity scores and food intake $[43,66,68]$.

Many studies have shown that chronic pain can alter the DA and opioidergic systems. In rats, chronic inflammatory and neuropathic pain reduced morphine-induced conditioned place preference (CPP), increased the expression of kappa receptors in the $\mathrm{NAc}$, and downregulated mu receptors in the ventral tegmental area (VTA). These processes were combined with suppression of morphine-induced DA release in the NAc [69-71]. Hipólito et al. reported that CFA-induced inflammatory pain downregulated the expression of $\mathrm{mu}$ receptors in the VTA and reduced the baseline DA level in the NAc, which had the combined effect of reducing food SA behavior [62]. Human research also showed the mu receptors in patients with chronic pain were downregulated [72, 73].

Otherwise, findings from a recent animal study suggest that D2-like receptors are involved in reward-seeking behavior. Specific ablation of medium spiny neurons that express D2-like receptors (D2-MSNs) in the ventrolateral striatum reduced goal-directed behavior in mice, executing a three-choice serial reaction-time task [74]. Optogenetic inhibition of D2-MSNs before lever presentation in an SA model also decreased the BP under a PR protocol in rats [74]. Another recent study showed that the downregulation of D2-MSNs under chronic pain correlated with decreased reward-seeking motivation in mice; SNI and CFA injection inhibited the excitatory postsynaptic potential of D2-MSNs, but not that of D1-MSNs, in the NAc [61]. The authors also found that the food-seeking behavior of these animals was inhibited under a PR protocol, but not under an FR protocol; sucrose-containing water preference and daily food consumption were unchanged. Neuroimage results from human research also support that D2-like receptor was downregulated by chronic pain. A PET study showed decreased availability (dysfunction) of D2-like (D2 and D3) receptors in the ventral striatum in patients with chronic nonneuropathic back pain [75]. Similar observations were made in female patients with fibromyalgia [76].

"Wanting"- and "liking"-related brain structures have been reviewed by Robinson et al. [15]. They proposed that the "liking" and "wanting" brain systems overlapped in mesolimbic structures. Pharmacological disruption of NAc could decrease the "wanting" behaviors such as spontaneous food and water intake [41, 48, 66, 67]. Besides, Schwartz et al. have reported that the neuropathic pain could decrease the neural activities in the $\mathrm{mPFC}-\mathrm{NAc}$ pathway and suppress the reward-seeking behaviors in the pain avoidance-reward approach task in rats [63]. The above studies indicate the potential neural mechanism that mediate the inhibition of reward processing in chronic pain, including DA system, mu receptor, D2-like receptor, and $\mathrm{mPFC}-\mathrm{NAc}$ pathway; however, further experiments are still needed to explore these mechanisms.

\section{Limitations}

The main limitation of this study is related to the differences between animal and human studies of changes in rewardrelated behaviors and neural mechanisms. For example, Pool et al. argue that differences in the definitions of "wanting" and "liking," which are more conflicted in humans than in animal research, lead to the use of animal-based experimental designs that do not distinguish "wanting" and "liking" in humans [40]. Experimental results from animal research can provide reference, but could not equal with results from human research and could not apply the conclusion to human research. Systematic human research needs to be performed to acquire more evidence on reward processing in the context of chronic pain.

\section{Conclusion and Future Directions}

In this review, we employed incentive salience theory to describe evidence on reward processing under chronic pain. The distinction of "liking" and "wanting" components of reward processing may aid discrimination of the effects of chronic pain on pleasurable sensory input and rewardseeking motivation. Based on evidence accumulated to date, we conclude that chronic pain reduces "wanting" behaviors. Bursts of reward-seeking behavior involve cognitive processes such as attention, working memory, 
and associative memory retrieval. Patients with chronic pain are sensitized to pain-related information, and pain management occupies their attentional resources [77, 78]. As a result, long-term chronic pain-induced attentional impairment might conflict with reward-seeking behavior, leading to the adaptive reduction of "wanting." In addition, some studies have shown that chronic pain can decrease "liking" behaviors.

The effect of chronic pain on reward processing seems to differ between the sexes. Chronic pain has been found to decrease reward-related behaviors (i.e., sexual behavior and sucrose preference) in female, but not in male, animals $[79,80]$. Sexual hormones may mediate reward processing, given the higher prevalence of food addiction and drug abuse in female than in male rats [81]. In human research, females are also more vulnerable to chronic pain than males $[82,83]$. Future studies should thus explore sex differences in the vulnerability of reward processing to chronic pain.

The "liking" and "wanting" perspective may provide a new way of thinking about reward processing under chronic pain. First, according to this perspective, reward sensitivity and reward-seeking motivation can change without affecting each other. Therefore, this framework could explain fundamental research findings that chronic pain does not decrease the ability to discriminate pleasurable sensory input, but does reduce reward-seeking behavior [60, 61]. Second, this perspective can be used to examine the comorbidity of chronic pain and drug addiction. Incentive salience theory has been used widely to explain drug addiction in humans and animals; for example, some addictive drugs sensitize the "wanting" system, resulting in compulsive drug-seeking behavior $[15,81]$. Patients with chronic pain are more likely to have contact with analgesics such as morphine and heroin, increasing the risk of drug addiction. Third, this perspective may enhance targeted drug treatment to improve reward processing in patients with chronic pain, as beyond psychological dissociation, "wanting" and "liking" are distinct in terms of brain structure and neurochemistry. Finally, as chronic pain is a worldwide problem that severely compromises the quality of daily life, further research should be conducted to explore whether this perspective can be applied to improve the well-being of patients with chronic pain.

\section{Conflicts of Interest}

The authors declare that there are no conflicts of interest regarding the publication of this paper.

\section{Acknowledgments}

This work was funded by an NNSF (National Natural Science Foundation of China) grant (31671140) to N.W., an NNSF grant (31271092) to J.W., an NNSF grant (31471061) to F. L., grants from CAS Key Laboratory of Mental Health, Institute of Psychology (KLMH 2014G01 and KLMH2016K02), and a grant from the initiation fund of the CAS/SAFEA International Partnership Program for Creative Research Teams (Y2CX131003).

\section{References}

[1] L. T. S. Pina, D. N. Gouveia, J. S. Costa et al., "New perspectives for chronic pain treatment: a patent review (20102016)," Expert Opinion on Therapeutic Patents, vol. 27, no. 7, pp. 787-796, 2017.

[2] M. A. Ilgen, F. Kleinberg, R. V. Ignacio et al., "Noncancer pain conditions and risk of suicide," JAMA Psychiatry, vol. 70, no. 7, pp. 692-697, 2013.

[3] J. D. Loeser, "Relieving pain in America," The Clinical Journal of Pain, vol. 28, no. 3, pp. 185-186, 2012.

[4] H. Breivik, B. Collett, V. Ventafridda, R. Cohen, and D. Gallacher, "Survey of chronic pain in Europe: prevalence, impact on daily life, and treatment," European Journal of Pain, vol. 10, no. 4, p. 287, 2006.

[5] W. Jackson, E. L. Zale, S. J. Berman et al., "Physical functioning and mindfulness skills training in chronic pain: a systematic review," Journal of Pain Research, vol. 12, pp. 179-189, 2019.

[6] W. Schultz, "Dopamine signals for reward value and risk: basic and recent data," Behavioral and Brain Functions, vol. 6, no. 1, p. 24, 2010.

[7] D. H. Zald and M. T. Treadway, "Reward processing, neuroeconomics, and psychopathology," Annual Review of Clinical Psychology, vol. 13, no. 1, pp. 471-495, 2017.

[8] A. E. Whitton, M. T. Treadway, and D. A. Pizzagalli, "Reward processing dysfunction in major depression, bipolar disorder and schizophrenia," Current Opinion in Psychiatry, vol. 28, no. 1, pp. 7-12, 2015.

[9] P. Karoly, M. A. Okun, C. Enders, and H. Tennen, "Effects of pain intensity on goal schemas and goal pursuit: a daily diary study," Health Psychology, vol. 33, no. 9, pp. 968-976, 2014.

[10] C. J. Mun, P. Karoly, and M. A. Okun, "Effects of daily pain intensity, positive affect, and individual differences in pain acceptance on work goal interference and progress," Pain, vol. 156, no. 11, pp. 2276-2285, 2015.

[11] P. Geha, I. de Araujo, B. Green, and D. M. Small, "Decreased food pleasure and disrupted satiety signals in chronic low back pain," Pain, vol. 155, no. 4, pp. 712-722, 2014.

[12] C. Chen, T. Takahashi, S. Nakagawa, T. Inoue, and I. Kusumi, "Reinforcement learning in depression: a review of computational research," Neuroscience \& Biobehavioral Reviews, vol. 55, pp. 247-267, 2015.

[13] S. J. Rizvi, D. A. Pizzagalli, B. A. Sproule, and S. H. Kennedy, "Assessing anhedonia in depression: potentials and pitfalls," Neuroscience \& Biobehavioral Reviews, vol. 65, pp. 21-35, 2016.

[14] L. Pizzagalli, C. E. Waugh, and I. H. Gotlib, "Anticipatory pleasure predicts motivation for reward in major depression," Journal of Abnormal Psychology, vol. 121, no. 1, pp. 51-60, 2012.

[15] M. J. Robinson, A. M. Fischer, A. Ahuja, E. N. Lesser, and H. Maniates, "Roles of "wanting" and "liking" in motivating behavior: gambling, food, and drug addictions," Behavioral Neuroscience of Motivation, vol. 27, pp. 105-136, 2016.

[16] W. Gandhi, S. Becker, and P. Schweinhardt, "Pain increases motivational drive to obtain reward, but does not affect associated hedonic responses: a behavioural study in healthy volunteers," European Journal of Pain, vol. 17, no. 7, pp. 1093-1103, 2013.

[17] K. E. Darbor, H. C. Lench, and A. R. Carter-Sowell, "Do people eat the pain away? The effects of acute physical pain on subsequent consumption of sweet-tasting food," PLoS One, vol. 11, no. 11, Article ID e0166931, 2016. 
[18] D. M. Small and A. V. Apkarian, "Increased taste intensity perception exhibited by patients with chronic back pain," Pain, vol. 120, no. 1-2, pp. 124-130, 2006.

[19] R. Nusslock and L. B. Alloy, "Reward processing and moodrelated symptoms: an RDoC and translational neuroscience perspective," Journal of Affective Disorders, vol. 216, pp. 3-16, 2017.

[20] R. Alloy and D. A. Pizzagalli, "Dysfunctional reward processing in depression," Current Opinion in Psychology, vol. 4, pp. 114-118, 2015.

[21] J. J. Marbach and P. Lund, "Depression, anhedonia and anxiety in temporomandibular joint and other facial pain syndromes," Pain, vol. 11, no. 1, pp. 73-84, 1981.

[22] J. J. Marbach, D. M. Richlin, and J. A. Lipton, "Illness behavior, depression and anhedonia in myofascial face and back pain patients," Psychotherapy and Psychosomatics, vol. 39, no. 1, pp. 47-54, 1983.

[23] C. Carver and T. L. White, "Behavioral inhibition, behavioral activation, and affective responses to impending reward and punishment: the BIS/BAS Scales," Journal of Personality and Social Psychology, vol. 67, no. 2, pp. 319-333, 1994.

[24] J. A. Becerra-García and M. J. Robles Jurado, "Behavioral approach system activity and self-reported somatic symptoms in fibromyalgia: an exploratory study," International Journal of Rheumatic Diseases, vol. 17, no. 1, pp. 89-92, 2014.

[25] N. A. Elvemo, N. I. Landrø, P. C. Borchgrevink, and A. K. Håberg, "Reward responsiveness in patients with chronic pain," European Journal of Pain, vol. 19, no. 10, pp. 1537-1543, 2015.

[26] J. T. Landrø and J. B. Vancouver, "Goal constructs in psychology: structure, process, and content," Psychological Bulletin, vol. 120, no. 3, pp. 338-375, 1996.

[27] E. Vancouver and T. M. Palermo, "Goal pursuit in youth with chronic pain," Children (Basel), vol. 3, no. 4, 2016.

[28] N. Claes, J. W. S. Vlaeyen, E. Lauwerier, M. Meulders, and G. Crombez, "Goal conflict in chronic pain: day reconstruction method," PeerJ, vol. 6, article e5272, 2018.

[29] E. Fisher, E. Keogh, and C. Eccleston, "Adolescents' approachavoidance behaviour in the context of pain," Pain, vol. 157, no. 2, pp. 370-376, 2016.

[30] A. H. Threadgill and P. A. Gable, "The sweetness of successful goal pursuit: approach-motivated pregoal states enhance the reward positivity during goal pursuit," International Journal of Psychophysiology, vol. 132, pp. 277-286, 2018.

[31] A. H. Threadgill and P. A. Gable, "Approach-motivated pregoal states enhance the reward positivity," Psychophysiology, vol. 53, no. 5, pp. 733-738, 2016.

[32] J. K. Hardy, L. J. Crofford, and S. C. Segerstrom, "Goal conflict, distress, and pain in women with fibromyalgia: a daily diary study," Journal of Psychosomatic Research, vol. 70, no. 6, pp. 534-540, 2011.

[33] K. C. Berridge, "The debate over dopamine's role in reward: the case for incentive salience," Psychopharmacology, vol. 191, no. 3, pp. 391-431, 2007.

[34] K. C. Berridge, T. E. Robinson, and J. W. Aldridge, "Dissecting components of reward: "liking," "wanting," and learning," Current Opinion in Pharmacology, vol. 9, no. 1, pp. 65-73, 2009.

[35] R. A. Wise, "Dopamine and reward: the anhedonia hypothesis 30 years on," Neurotoxicity Research, vol. 14, no. 2-3, pp. 169-183, 2008.

[36] K. C. Berridge and T. E. Robinson, "What is the role of dopamine in reward: hedonic impact, reward learning, or incentive salience?," Brain Research Reviews, vol. 28, no. 3, pp. 309-369, 1998.
[37] T. E. Robinson and K. C. Berridge, "Addiction," Annual Review of Psychology, vol. 54, no. 1, pp. 25-53, 2003.

[38] M. Cabanac, "Physiological role of pleasure," Science, vol. 173, no. 4002, pp. 1103-1107, 1971.

[39] R. A. Wise, "Dopamine, learning and motivation," Nature Reviews Neuroscience, vol. 5, no. 6, pp. 483-494, 2004.

[40] E. Pool, V. Sennwald, S. Delplanque, T. Brosch, and D. Sander, "Measuring wanting and liking from animals to humans: a systematic review," Neuroscience \& Biobehavioral Reviews, vol. 63, pp. 124-142, 2016.

[41] S. Robinson, S. M. Sandstrom, V. H. Sandstrom, and R. D. Palmiter, "Distinguishing whether dopamine regulates liking, wanting, and/or learning about rewards," Behavioral Neuroscience, vol. 119, no. 1, pp. 5-15, 2005.

[42] K. C. Berridge, "Food reward: brain substrates of wanting and liking," Neuroscience \& Biobehavioral Reviews, vol. 20, no. 1, pp. 1-25, 1996.

[43] D. C. Castro and K. C. Berridge, "Opioid hedonic hotspot in nucleus accumbens shell: mu, delta, and kappa maps for enhancement of sweetness "liking" and "wanting", "Journal of Neuroscience, vol. 34, no. 12, pp. 4239-4250, 2014.

[44] S. E. Ross, E. Levin, C. A. Itoga, C. B. Schoen, R. Selmane, and J. W. Aldridge, "Deep brain stimulation in the central nucleus of the amygdala decreases "wanting" and "liking" of food rewards," European Journal of Neuroscience, vol. 44, no. 7, pp. 2431-2445, 2016.

[45] J. W. de Jong, T. J. M. Roelofs, F. M. U. Mol et al., "Reducing ventral tegmental dopamine D2 receptor expression selectively boosts incentive motivation," Neuropsychopharmacology, vol. 40, no. 9, pp. 2085-2095, 2015.

[46] S. L. Robinson and B. A. McCool, "Microstructural analysis of rat ethanol and water drinking patterns using a modified operant self-administration model," Physiology \& Behavior, vol. 149, pp. 119-130, 2015.

[47] D. C. Castro and K. C. Berridge, "Opioid and orexin hedonic hotspots in rat orbitofrontal cortex and insula," Proceedings of the National Academy of Sciences, vol. 114, no. 43, pp. 9125-9134, 2017.

[48] S. Peciña, B. Cagniard, K. C. Berridge, J. W. Aldridge, and $\mathrm{X}$. Zhuang, "Hyperdopaminergic mutant mice have higher "wanting" but not "liking" for sweet rewards," The Journal of Neuroscience, vol. 23, no. 28, pp. 9395-9402, 2003.

[49] A. Dellarole, P. Morton, R. Brambilla et al., "Neuropathic paininduced depressive-like behavior and hippocampal neurogenesis and plasticity are dependent on TNFR1 signaling," Brain, Behavior, and Immunity, vol. 41, pp. 65-81, 2014.

[50] J. Wang, Y. Goffer, D. Xu et al., "A single subanesthetic dose of ketamine relieves depression-like behaviors induced by neuropathic pain in rats," Anesthesiology, vol. 115, no. 4, pp. 812-821, 2011.

[51] J. Wu, Z. Zhao, B. Sabirzhanov et al., "Spinal cord injury causes brain inflammation associated with cognitive and affective changes: role of cell cycle pathways," Journal of Neuroscience, vol. 34, no. 33, pp. 10989-11006, 2014.

[52] A. S. Bura, T. Guegan, D. Zamanillo, J. M. Vela, and R. Maldonado, "Operant self-administration of a sigma ligand improves nociceptive and emotional manifestations of neuropathic pain," European Journal of Pain, vol. 17, no. 6, pp. 832-843, 2013.

[53] Y.-T. Liu, Y.-W. Shao, C.-T. Yen, and F.-Z. Shaw, "Acidinduced hyperalgesia and anxio-depressive comorbidity in rats," Physiology \& Behavior, vol. 131, pp. 105-110, 2014.

[54] M. Shi, W.-J. Qi, G. Gao, J.-Y. Wang, and F. Luo, "Increased thermal and mechanical nociceptive thresholds in rats with 
depressive-like behaviors," Brain Research, vol. 1353, pp. 225-233, 2010.

[55] M. Shi, J.-Y. Wang, and F. Luo, "Depression shows divergent effects on evoked and spontaneous pain behaviors in rats," The Journal of Pain, vol. 11, no. 3, pp. 219-229, 2010.

[56] C. Su, J. D’amour, M. Lee et al., "Persistent pain alters AMPA receptor subunit levels in the nucleus accumbens," Molecular Brain, vol. 8, p. 46, 2015.

[57] R. Urban, G. Scherrer, E. H. Goulding, L. H. Tecott, and A. I. Basbaum, "Behavioral indices of ongoing pain are largely unchanged in male mice with tissue or nerve injury-induced mechanical hypersensitivity," Pain, vol. 152, no. 5, pp. 9901000, 2011.

[58] L. Bravo, J. A. Mico, R. Rey-Brea, B. Pérez-Nievas, J. C. Leza, and E. Berrocoso, "Depressive-like states heighten the aversion to painful stimuli in a rat model of comorbid chronic pain and depression," Anesthesiology, vol. 117, no. 3, pp. 613-625, 2012.

[59] Y. Goffer, D. Xu, S. E. Eberle et al., "Calcium-permeable AMPA receptors in the nucleus accumbens regulate depression-like behaviors in the chronic neuropathic pain state," Journal of Neuroscience, vol. 33, no. 48, pp. 1903419044, 2013.

[60] A. Okun, D. L. McKinzie, J. M. Witkin et al., "Hedonic and motivational responses to food reward are unchanged in rats with neuropathic pain," Pain, vol. 157, no. 12, pp. 2731-2738, 2016.

[61] N. Schwartz, P. Temkin, S. Jurado et al., "Decreased motivation during chronic pain requires long-term depression in the nucleus accumbens," Science, vol. 345, no. 6196, pp. 535-542, 2014.

[62] L. Hipolito, A. Wilson-Poe, Y. Campos-Jurado et al., "Inflammatory pain promotes increased opioid selfadministration: role of dysregulated ventral tegmental area opioid receptors," Journal of Neuroscience, vol. 35, no. 35, pp. 12217-12231, 2015.

[63] N. Schwartz, C. Miller, and H. L. Fields, "Cortico-Accumbens regulation of approach-avoidance behavior is modified by experience and chronic pain," Cell Reports, vol. 19, no. 8, pp. 1522-1531, 2017.

[64] D. Amorim, A. David-Pereira, A. Pertovaara, A. Almeida, and F. Pinto-Ribeiro, "Amitriptyline reverses hyperalgesia and improves associated mood-like disorders in a model of experimental monoarthritis," Behavioural Brain Research, vol. 265, pp. 12-21, 2014.

[65] W. Hodos, "Progressive ratio as a measure of reward strength,” Science, vol. 134, no. 3483, pp. 943-944, 1961.

[66] S. Peciña and K. C. Berridge, "Dopamine or opioid stimulation of nucleus accumbens similarly amplify cue-triggered "wanting" for reward: entire core and medial shell mapped as substrates for PIT enhancement," European Journal of Neuroscience, vol. 37, no. 9, pp. 1529-1540, 2013.

[67] C. M. Cannon and R. D. Palmiter, "Reward without dopamine," The Journal of Neuroscience, vol. 23, no. 34, pp. 10827-10831, 2003.

[68] K. S. Smith, K. C. Berridge, and J. W. Aldridge, "Disentangling pleasure from incentive salience and learning signals in brain reward circuitry," Proceedings of the National Academy of Sciences, vol. 108, no. 27, pp. E255-E264, 2011.

[69] M. Narita, Y. Kishimoto, Y. Ise, Y. Yajima, K. Misawa, and T. Suzuki, "Direct evidence for the involvement of the mesolimbic $\kappa$-opioid system in the morphine-induced rewarding effect under an inflammatory pain-like state," Neuropsychopharmacology, vol. 30, no. 1, pp. 111-118, 2005.
[70] S. Ozaki, M. Narita, M. Narita et al., "Suppression of the morphine-induced rewarding effect in the rat with neuropathic pain: implication of the reduction in $\mu$-opioid receptor functions in the ventral tegmental area," Journal of Neurochemistry, vol. 82, no. 5, pp. 1192-1198, 2002.

[71] K. Niikura, M. Narita, M. Narita et al., "Direct evidence for the involvement of endogenous $\beta$-endorphin in the suppression of the morphine-induced rewarding effect under a neuropathic pain-like state," Neuroscience Letters, vol. 435, no. 3, pp. 257-262, 2008.

[72] R. E. Harris, D. J. Clauw, D. J. Scott, S. A. McLean, R. H. Gracely, and J.-K. Zubieta, "Decreased central-opioid receptor availability in fibromyalgia," Journal of Neuroscience, vol. 27, no. 37, pp. 10000-10006, 2007.

[73] A. Schrepf, D. E. Harper, S. E. Harte et al., "Endogenous opioidergic dysregulation of pain in fibromyalgia," Pain, vol. 157, no. 10, pp. 2217-2225, 2016.

[74] I. Tsutsui-Kimura, H. Takiue, K. Yoshida et al., "Dysfunction of ventrolateral striatal dopamine receptor type 2-expressing medium spiny neurons impairs instrumental motivation," Nature Communications, vol. 8, p. 14304, 2017.

[75] I. K. Martikainen, E. B. Nuechterlein, M. Pecina et al., "Chronic back pain is associated with alterations in dopamine neurotransmission in the ventral striatum," Journal of $\mathrm{Neu}$ roscience, vol. 35, no. 27, pp. 9957-9965, 2015.

[76] D. S. Albrecht, P. J. MacKie, D. A. Kareken et al., "Differential dopamine function in fibromyalgia," Brain Imaging and Behavior, vol. 10, no. 3, pp. 829-839, 2016.

[77] G. Crombez, D. M. L. Ryckeghem, C. Van Ryckeghem, and S. Van Damme, "Attentional bias to pain-related information: a meta-analysis," Pain, vol. 154, no. 4, pp. 497-510, 2013.

[78] C. Berryman, T. R. Stanton, J. K. Bowering, A. Tabor, A. McFarlane, and L. G. Moseley, "Evidence for working memory deficits in chronic pain: a systematic review and meta-analysis," Pain, vol. 154, no. 8, pp. 1181-1196, 2013.

[79] M. A. Farmer, A. Leja, E. Foxen-Craft et al., "Pain reduces sexual motivation in female but not male mice," Journal of Neuroscience, vol. 34, no. 17, pp. 5747-5753, 2014.

[80] J. Luo, T. Wang, S. Liang, X. Hu, W. Li, and F. Jin, "Experimental gastritis leads to anxiety- and depression-like behaviors in female but not male rats," Behavioral and Brain Functions, vol. 9, no. 1, p. 46, 2013.

[81] K. K. Pitchers, S. B. Flagel, E. G. O’Donnell, L. C. Solberg Woods, M. Sarter, and T. E. Robinson, "Individual variation in the propensity to attribute incentive salience to a food cue: influence of sex," Behavioural Brain Research, vol. 278, pp. 462-469, 2015.

[82] R. M. Craft, J. S. Mogil, and A. M. Aloisi, "Sex differences in pain and analgesia: the role of gonadal hormones," European Journal of Pain, vol. 8, no. 5, pp. 397-411, 2004.

[83] O. van Hecke, N. Torrance, and B. H. Smith, "Chronic pain epidemiology and its clinical relevance," British Journal of Anaesthesia, vol. 111, no. 1, pp. 13-18, 2013. 


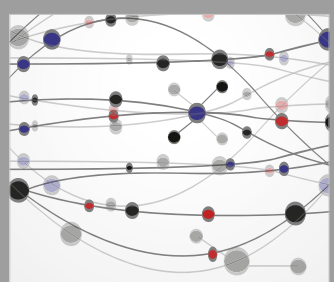

The Scientific World Journal
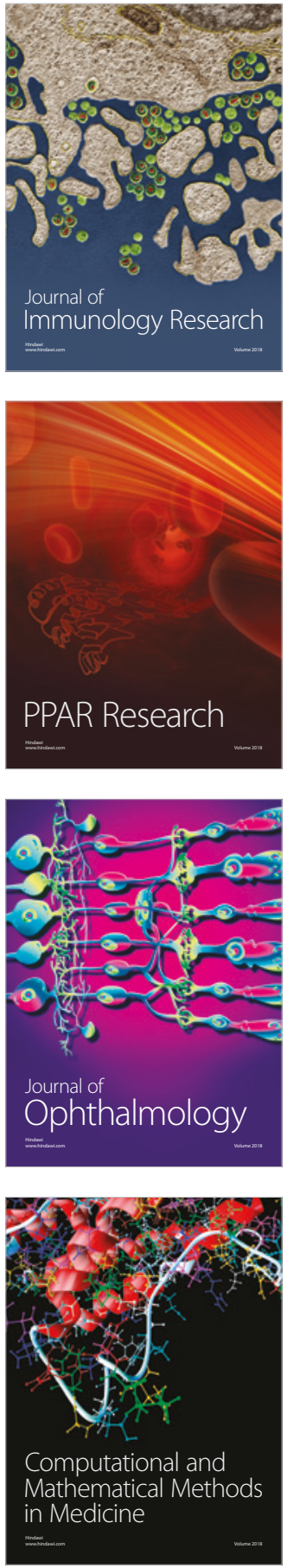

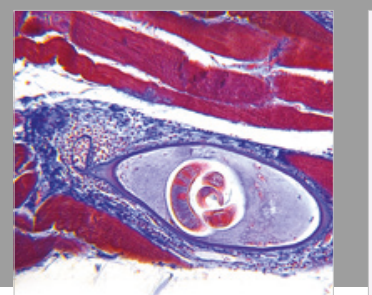

Gastroenterology Research and Practice

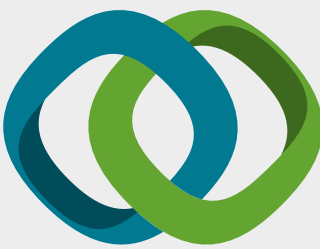

\section{Hindawi}

Submit your manuscripts at

www.hindawi.com
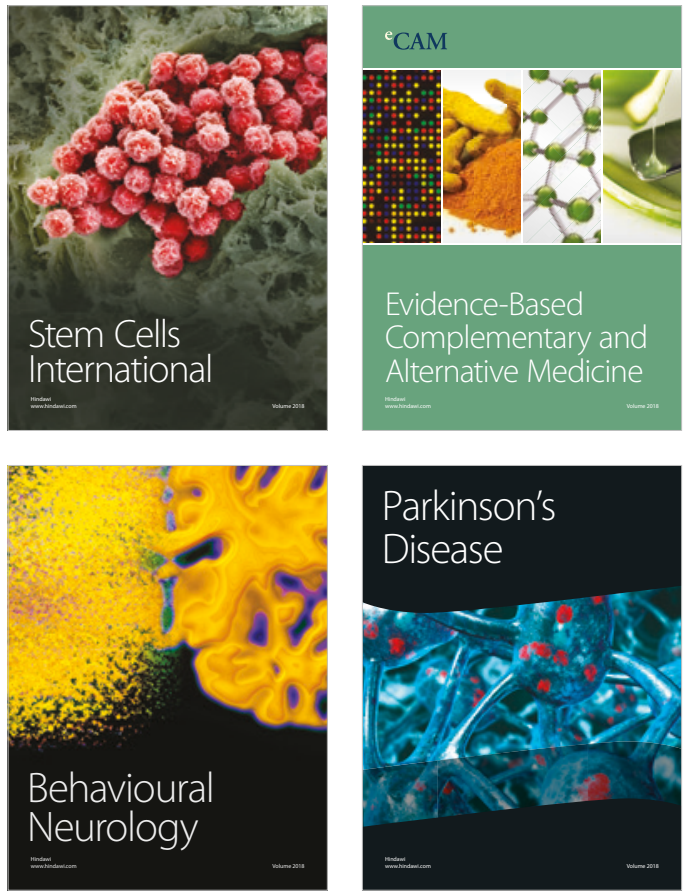

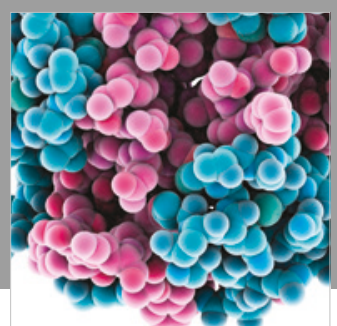

ournal of

Diabetes Research

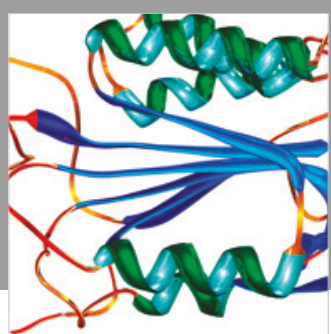

Disease Markers
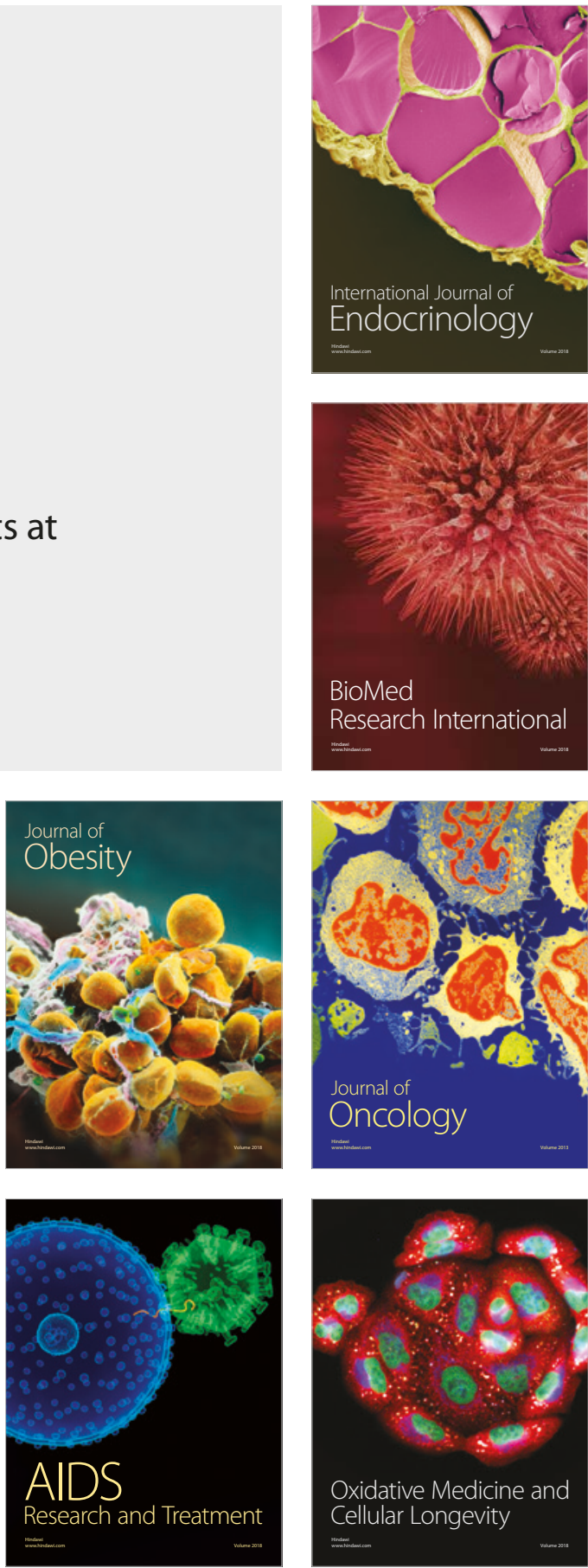